\title{
TERMS WHICH ARE MAL'CEV MODULO SOME FUNCTIONS
}

\author{
PAOLO LIPPARINI
}

\begin{abstract}
We derive consequences from the existence of a term which satisfies Mal'cev identities (characterizing permutability) modulo two functions $F$ and $G$ from admissible relations to admissible relations. We also provide characterizations of varieties having a Mal'cev term modulo $F$ and $G$.
\end{abstract}

\section{INTRODUCTION}

In 1954 A. I. Mal'cev [16] proved the following fundamental result.

Theorem 1.1. All algebras in a variety $\mathcal{V}$ have permutable congruences if and only if $\mathcal{V}$ has a ternary term $t$ such that the following identities are valid in $\mathcal{V}$ :

$$
x=t(x, y, y) \quad t(x, x, y)=y .
$$

Mal'cev's Theorem 1.1 can be safely considered the beginning of modern Universal Algebra. On one side, it has initiated the study of what are nowadays known as Mal'cev conditions. See, e.g., [5, 1] for information and further references. On the other side, it has eventually led to a more and more refined theory of permutable varieties, culminating perhaps in J. D. H. Smith's commutator theory [17].

While generalizing Smith's theory to the congruence modular case, many researchers independently discovered that congruence modular varieties have a difference term, a term which satisfies Mal'cev identities, except that one equality is replaced by congruence modulo the commutator (the commutator $[\alpha, \beta]$ of two congruences can be defined in many ways, but the actual definition is not relevant to the present paper). In more detail, a difference term is a term satisfying the conditions $a=t(a, b, b)$ and $t(a, a, b)[\alpha, \alpha] b$, whenever $a \alpha b$. Notice that, for notational convenience, if $R$ is a binary relation, we shall usually write $a R b$ in place of $(a, b) \in R$.

The difference term has found a great deal of applications in the theory of congruence modular varieties; see the survey books by H-P. Gumm [3],

Date: Put on the author's web page March 12, 2006.

2000 Mathematics Subject Classification. Primary 08A99; 08B99.

Key words and phrases. Commutator, congruence, admissible relation; (weak) difference term, term Mal'cev modulo two functions.

The author has received support from MPI and GNSAGA. 
and R. Freese and R. McKenzie [2] for some history. See, e.g., [1, 6, 12, 13] and references there for more recent results.

When commutator theory for congruence modular varieties was at its beginning, the general feeling was that the theory could not be generalized to a larger setting. However, already in [18], W. Taylor proved that abelian algebras are affine in $m$-permutable varieties, a result previously known to hold in the case of modular varieties. W. Taylor worried about the possibility that his result might be dependent on the definition of the commutator, but later it has been shown in [9, Corollary 5.9] that many definitions of "abelian" coincide in a large class of varieties, including $m$-permutable ones.

More importantly, the proof of [18, Theorem 2] actually shows that every $m$-permutable variety has a weak difference term, that is, a term which satisfies the form of Mal'cev identities in which both equalities are replaced by congruence modulo the commutator. More explicitly, a weak difference term is a term satisfying $a[\alpha, \alpha] t(a, b, b)$ and $t(a, a, b)[\alpha, \alpha] b$, whenever $a \alpha b$. Many conditions equivalent to the existence of a (weak) difference term have been given in [12]. Though $m$-permutable varieties do not have, in general, a difference term, the weak difference term is enough for many applications; for example, Taylor's result can be used to get a rather simple proof that congruence lattices of algebras in $m$-permutable varieties satisfy non trivial identities; see $[10,11,15]$.

Taylor's Theorem is probably the first significant result in commutator theory for non modular varieties, but the theory was ready for successful developments. When D. Hobby and R. McKenzie [4] provided their fundamental classification of locally finite varieties, commutators and centralizers played an important role in it (let us mention that, meanwhile, many results from [4] have been extended from locally finite varieties to arbitrary varieties. K. Kearnes and E. Kiss have announced [8] a book on the subject). Weak difference terms, too, under a different terminology, played a role in Chapters 7 and 9 of [4]. In particular, [4, Theorem 9.6] implies that a locally finite variety omits type $\mathbf{1}$ if and only if it has a weak difference term.

Motivated by the above results from $[4,18]$, we begun in [9] a study of algebras and varieties with a weak difference term for their own sake. We showed that a large part of the commutator theory for congruence modular varieties follows just from the assumption of the existence of a difference term (see also $[6,13])$. More surprisingly, a weak difference term is enough for a large amount of results. As a by-product of our study, in [9] we succeeded in generalizing to arbitrary varieties some results first proved by Hobby and McKenzie for locally finite varieties only. Actually, [9] provided further results which were new even in the particular case of locally finite varieties.

In addition, Section 3 in [9] deals with an even more general situation: we only assume that $F, G: \operatorname{Con}(\mathbf{A}) \rightarrow \operatorname{Con}(\mathbf{A})$, and study terms which are Mal'cev modulo $F$ and $G$, called there $F$-G-difference terms. In this sense, a difference term is a term Mal'cev modulo $F(\alpha)=0$ constantly, and $G(\alpha)=$ 
$[\alpha, \alpha]$; and a weak difference term is a term Mal'cev modulo $F(\alpha)=G(\alpha)=$ $[\alpha, \alpha]$. Thus, a unified treatment of both cases is accomplished; moreover, the theory becomes independent from the definition of the commutator. Further, on $[9$, p. 186], we mention the possibility that $\operatorname{Con}(\mathbf{A})$ might be replaced by $\operatorname{Adm}(\mathbf{A})$, the set of reflexive and admissible relations on A. The possibility of such a generalization appears particularly interesting because, in general, the proof that a variety $\mathcal{V}$ has a difference term, or a weak difference term, actually shows that $\mathcal{V}$ has a term Mal'cev modulo certain functions $F$ and $G$, whose images range over admissible relations, usually smaller than congruences. An example of this fact is provided in [10, Remark 3.5] for $m$-permutable varieties, and a similar remark holds for modular varieties. We shall describe in a sequel to the present paper applications of the above remarks.

Moreover, the results proved in $[7,14]$ suggest the possibility that an interesting commutator theory can be developed, dealing with reflexive and admissible relations, rather than congruences. In fact, $[14$, Part II $]$ provides characterizations of what might be called neutral varieties in the sense of commutators for relations. Though a full theory of commutators for relations goes beyond the scope of the present paper, we show that many results proved for algebras with a weak difference term (that is, dealing with congruences) generalize to the case of a term Mal'cev modulo two functions whose domains and codomains are just admissible and reflexive relations.

In particular, we generalize results from [9, Section 3] to the case of the existence of terms which are Mal'cev modulo $F, G: \operatorname{Adm}(\mathbf{A}) \rightarrow \operatorname{Adm}(\mathbf{A})$. In fact, some of the results proved here are stronger than the corresponding results in [9] even in the particular case $F, G: \operatorname{Con}(\mathbf{A}) \rightarrow \operatorname{Con}(\mathbf{A})$. For example, Lemma 2.1(i) here, though apparently simple, is a significant improvement of the case $n=1$ of [9, Lemma 3.1(i)] (we shall deal with the case $n>1$ in a sequel to the present paper). Lemma 2.1(i) is then used in order to prove Theorem 2.2 and Corollary 2.3(i), which give a description of $R_{1} \circ R_{2} \circ \cdots \circ R_{n}$ and provide, even for admissible relations, finer bounds than the ones obtained in [9, Lemma 3.1(iii)] for congruences. Moreover, in Corollary 2.3(viii)(x) we can compute the congruence generated by a relation in a more effective way than in [9, Proposition 3.7].

In Section 3 we deal with global operators defined on all algebras in a given variety $\mathcal{V}$. Many conditions equivalent to the existence of a term Mal'cev modulo $F$ and $G$ for all algebras in $\mathcal{V}$ are given, under the assumption that $F$ and $G$ satisfy a homomorphism property. Some results from [12, Part II] are particular cases of the results proved in Section 3. At the end of the section we show that all the commutators for relations introduced in [14] satisfy the required homomorphism property.

The main notion of the present paper is contained in the following definition. 
Definition 1.2. If $\mathbf{A}$ is an algebra, and $F: \operatorname{Adm}(\mathbf{A}) \rightarrow \operatorname{Adm}(\mathbf{A}), G:$ $\operatorname{Adm}(\mathbf{A}) \rightarrow \operatorname{Adm}(\mathbf{A})$, we say that a ternary term $t$ of $\mathbf{A}$ is Mal'cev modulo $F$ and $G$ if and only if

$$
a F(R) t(a, b, b) \quad \text { and } \quad t(a, a, b) G(R) b,
$$

whenever $a, b \in \mathbf{A}, R \in \operatorname{Adm}(\mathbf{A})$, and $a R b$.

An alternative name for the above notion is a weak difference term modulo $F$ and $G$, or simply an $F$-G-difference term: we used this terminology in [9, p. 199], in the case when $F: \operatorname{Con}(\mathbf{A}) \rightarrow \operatorname{Con}(\mathbf{A}), G: \operatorname{Con}(\mathbf{A}) \rightarrow \operatorname{Con}(\mathbf{A})$.

Notice that if $t(x, y, z)$ is Mal'cev modulo $F$ and $G$, then $t^{\prime}$ defined by $t^{\prime}(x, y, z)=t(z, y, x)$ is Mal'cev modulo $F^{\prime}$ and $G^{\prime}$, where $F^{\prime}$ and $G^{\prime}$ are defined by $F^{\prime}(R)=\left(G\left(R^{-}\right)\right)^{-}$and $G^{\prime}(R)=\left(F\left(R^{-}\right)\right)^{-}$.

We now fix notations. For a binary relation $R$ on some algebra, $R^{-}$ denotes the converse of $R, R^{*}$ is the transitive closure of $R$, and $C g(R)$ is the smallest congruence containing $R . \bar{R}$ denotes the least admissible relation containing $R$. Notice that if $R$ and $S$ are admissible, then $R \circ S$ is admissible, too. Intersection is denoted by juxtaposition. $R \circ_{n} S$ is $R \circ S \circ R \circ S \ldots$ with $n-1$ occurrences of $\circ$. $R^{n}=R \circ_{n} R$. By convention, we put $R^{0}=0$, where 0 denotes the identity relation (the smallest reflexive relation) on the given algebra; the largest binary relation is denoted by $1 . R+S=\bigcup_{n \in \omega} R \circ_{n} S$. We denote $n$-tuples by a bar, as in $\bar{a}$.

\section{Formulae For Sums}

Lemma 2.1. Suppose that $\mathbf{A}$ has a term Mal'cev modulo $F$ and $G$. Then for all reflexive admissible relations $R, S \in \operatorname{Adm}(\mathbf{A})$, and for arbitrary relations $\theta, \theta_{1}, \theta_{2} \subseteq A^{2}$, the following hold:

(i) If $a, b, c, d \in \mathbf{A}, a R b \theta_{1} c, a \theta_{2} d S c$, and $b \theta d$ (that is, the situation pictured in the following diagram occurs)

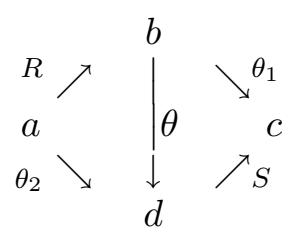

then $(a, c) \in F(R) \circ \overline{\theta_{2} \cup \theta \cup \theta_{1}} \circ G(S)$.

(ii) $R \circ \theta \circ S \subseteq F(R) \circ \overline{(R \circ \theta) \cup(\theta \circ S)} \circ G(S)$.

(iii) $R \circ S \subseteq F(R) \circ \overline{R \cup S} \circ G(S) \subseteq F(R) \circ S \circ R \circ G(S)$.

(iv) $R \circ R \subseteq F(R) \circ R \circ G(R)$.

Proof. (i) $a F(R) t(a, b, b) \overline{\theta_{2} \cup \theta \cup \theta_{1}} t(d, d, c) G(S) c$.

(ii) If $a R b \theta d S c$ then $a R b \theta d$ and $b \theta d S c$. Letting $\theta_{2}=R \circ \theta$ and $\theta_{1}=\theta \circ S$ we get the conclusion from (i), noticing that $\theta \subseteq R \circ \theta \subseteq \theta_{2}$, since $R$ is reflexive. 
The first inclusion in (iii) is the particular case $\theta=0$ of (ii). The second inclusion is trivial, since $R$ and $S$ are reflexive, and $S \circ R$ is admissible.

(iv) is the particular case $S=R$ of (iii).

By applying Lemma 2.1(i), we can generalize clause 2.1(iii) in order to get a formula for computing $R_{1} \circ R_{2} \circ \cdots \circ R_{n}$.

Theorem 2.2. Suppose that $\mathbf{A}$ has a term Mal'cev modulo $F$ and $G$. Then for all $n \geq 2$, and for all reflexive admissible relations $R_{1}, R_{2}, \ldots, R_{n} \in$ $\operatorname{Adm}(\mathbf{A})$, the following hold:

$$
\begin{aligned}
& R_{1} \circ R_{2} \circ \cdots \circ R_{n} \subseteq \\
& \quad F\left(R_{1}\right) \circ \overline{F\left(R_{1}\right) \cup F\left(R_{2}\right)} \circ \overline{F\left(R_{1}\right) \cup F\left(R_{2}\right) \cup F\left(R_{3}\right)} \circ \ldots \\
& \circ \overline{F\left(R_{1}\right) \cup F\left(R_{2}\right) \cup \cdots \cup F\left(R_{n-2}\right)} \circ \overline{F\left(R_{1}\right) \cup F\left(R_{2}\right) \cup \cdots \cup F\left(R_{n-1}\right)} \circ \\
& \overline{R_{1} \cup R_{2} \cup \cdots \cup R_{n-1} \cup R_{n} \circ} \\
& \overline{G\left(R_{2}\right) \cup G\left(R_{3}\right) \cup \cdots \cup G\left(R_{n}\right)} \circ \overline{G\left(R_{3}\right) \cup G\left(R_{4}\right) \cup \cdots \cup G\left(R_{n}\right)} \circ \ldots \\
& \circ \overline{G\left(R_{n-2}\right) \cup G\left(R_{n-1}\right) \cup G\left(R_{n}\right)} \circ \overline{G\left(R_{n-1}\right) \cup G\left(R_{n}\right)} \circ G\left(R_{n}\right) .
\end{aligned}
$$

Proof. The theorem is proved by induction on $n$.

The basis $n=2$ is given by Lemma 2.1(iii).

Suppose that the Theorem holds for some $n$, and that $\left(a_{0}, a_{n+1}\right) \in R_{1} \circ R_{2} \circ$ $\cdots \circ R_{n} \circ R_{n+1}$. This means that there are further elements $a_{i}(1 \leq i \leq n)$ such that $a_{0} R_{1} a_{1} R_{2} a_{2} \ldots a_{n-2} R_{n-1} a_{n-1} R_{n} a_{n} R_{n+1} a_{n+1}$. This implies that $\left(a_{0}, a_{n}\right) \in R_{1} \circ R_{2} \circ \cdots \circ R_{n}$. If we denote by $\theta_{2}$ the right-hand side of the formula in the statement of Theorem 2.2, we get, by the inductive assumption, $R_{1} \circ R_{2} \circ \cdots \circ R_{n} \subseteq \theta_{2}$, hence $a_{0} \theta_{2} a_{n}$.

Similarly, since $\left(a_{1}, a_{n+1}\right) \in R_{2} \circ \cdots \circ R_{n} \circ R_{n+1}$, and letting

$$
\begin{aligned}
& \theta_{1}= \\
& F\left(R_{2}\right) \circ \overline{F\left(R_{2}\right) \cup F\left(R_{3}\right)} \circ \overline{F\left(R_{2}\right) \cup F\left(R_{3}\right) \cup F\left(R_{4}\right)} \circ \ldots \\
& \circ \overline{F\left(R_{2}\right) \cup F\left(R_{3}\right) \cup \cdots \cup F\left(R_{n-1}\right)} \circ \overline{F\left(R_{2}\right) \cup F\left(R_{3}\right) \cup \cdots \cup F\left(R_{n}\right)} \circ \\
& \overline{R_{2} \cup R_{3} \cup \cdots \cup R_{n} \cup R_{n+1}} \text { ○ } \\
& \overline{G\left(R_{3}\right) \cup G\left(R_{4}\right) \cup \cdots \cup G\left(R_{n+1}\right)} \circ \overline{G\left(R_{4}\right) \cup G\left(R_{5}\right) \cup \cdots \cup G\left(R_{n+1}\right)} \circ \ldots \\
& \circ \overline{G\left(R_{n-1}\right) \cup G\left(R_{n}\right) \cup G\left(R_{n+1}\right)} \circ \overline{G\left(R_{n}\right) \cup G\left(R_{n+1}\right)} \circ G\left(R_{n+1}\right),
\end{aligned}
$$


we get, again by the inductive assumption, $R_{2} \circ \cdots \circ R_{n} \circ R_{n+1} \subseteq \theta_{1}$, hence $a_{1} \theta_{1} a_{n+1}$. Putting

$$
\begin{aligned}
& \Psi= \\
& \overline{F\left(R_{1}\right) \cup F\left(R_{2}\right) \cup F\left(R_{3}\right)} \circ \overline{F\left(R_{1}\right) \cup F\left(R_{2}\right) \cup F\left(R_{3}\right) \cup F\left(R_{4}\right)} \circ \cdots \\
& \circ \overline{F\left(R_{1}\right) \cup F\left(R_{2}\right) \cup \cdots \cup F\left(R_{n-1}\right)} \circ \overline{F\left(R_{1}\right) \cup F\left(R_{2}\right) \cup \cdots \cup F\left(R_{n}\right)} \circ \\
& \quad \overline{R_{1} \cup R_{2} \cup \cdots \cup R_{n-1} \cup R_{n} \cup R_{n+1}} \circ \\
& \hline \overline{G\left(R_{2}\right) \cup G\left(R_{3}\right) \cup \cdots \cup G\left(R_{n+1}\right)} \circ \overline{G\left(R_{3}\right) \cup G\left(R_{4}\right) \cup \cdots \cup G\left(R_{n+1}\right)} \circ \cdots \\
& \circ \overline{G\left(R_{n-2}\right) \cup \cdots \cup G\left(R_{n+1}\right)} \circ \overline{G\left(R_{n-1}\right) \cup G\left(R_{n}\right) \cup G\left(R_{n+1}\right)},
\end{aligned}
$$

we compute

$$
\theta_{2} \cup \theta_{1} \subseteq\left(F\left(R_{1}\right) \cup F\left(R_{2}\right)\right) \circ \Psi \circ\left(G\left(R_{n}\right) \cup G\left(R_{n+1}\right)\right),
$$

since $\left(\phi_{1} \circ \phi_{2} \circ \cdots \circ \phi_{m}\right) \cup\left(\xi_{1} \circ \xi_{2} \circ \cdots \circ \xi_{m}\right) \subseteq\left(\phi_{1} \cup \xi_{1}\right) \circ\left(\phi_{2} \cup \xi_{2}\right) \circ \cdots \circ\left(\phi_{m} \cup\right.$ $\left.\xi_{m}\right)$, for every $m$, and for arbitrary reflexive relations $\phi_{1}, \xi_{1}, \ldots$; and since, say, $\overline{F\left(R_{1}\right) \cup F\left(R_{2}\right)} \cup \overline{F\left(R_{2}\right) \cup F\left(R_{3}\right)} \subseteq \overline{F\left(R_{1}\right) \cup F\left(R_{2}\right) \cup F\left(R_{3}\right)}$. From the above-displayed formula we get

$$
\overline{\theta_{2} \cup \theta_{1}} \subseteq \overline{F\left(R_{1}\right) \cup F\left(R_{2}\right)} \circ \Psi \circ \overline{G\left(R_{n}\right) \cup G\left(R_{n+1}\right)},
$$

since the right-hand side is an admissible relation containing $\theta_{2} \cup \theta_{1}$. Letting $\theta=R_{2} \circ \cdots \circ R_{n}$, we have $a_{1} \theta a_{n}$. Thus we get from Lemma 2.1(i) with $a=a_{0}, b=a_{1}, d=a_{n}$ and $c=a_{n+1}$ :

$$
\left(a_{0}, a_{n+1}\right) \in F\left(R_{1}\right) \circ \overline{\theta_{2} \cup \theta \cup \theta_{1}} \circ G\left(R_{n+1}\right) .
$$

Notice that $\theta=R_{2} \circ \cdots \circ R_{n} \subseteq R_{2} \circ \cdots \circ R_{n} \circ R_{n+1} \subseteq \theta_{1}$, thus $\theta_{2} \cup \theta \cup \theta_{1}=\theta_{2} \cup \theta_{1}$. Summing up,

$$
\left(a_{0}, a_{n+1}\right) \in F\left(R_{1}\right) \circ \overline{F\left(R_{1}\right) \cup F\left(R_{2}\right)} \circ \Psi \circ \overline{G\left(R_{n}\right) \cup G\left(R_{n+1}\right)} \circ G\left(R_{n+1}\right) .
$$

We have proved

$R_{1} \circ R_{2} \circ \cdots \circ R_{n+1} \subseteq F\left(R_{1}\right) \circ \overline{F\left(R_{1}\right) \cup F\left(R_{2}\right)} \circ \Psi \circ \overline{G\left(R_{n}\right) \cup G\left(R_{n+1}\right)} \circ G\left(R_{n+1}\right)$, which is the statement of the theorem for $n+1$.

Corollary 2.3. Suppose that $\mathbf{A}$ has a term Mal'cev modulo $F$ and $G$. Then for all reflexive admissible relations $R, S, R_{1}, R_{2}, \cdots \in \operatorname{Adm}(\mathbf{A})$, the following inclusions hold for every $n \geq 0$ :

(i) $R \circ_{n+2} S \subseteq F(R) \circ(\overline{F(R) \cup F(S)})^{n} \circ \overline{R \cup S} \circ(\overline{G(R) \cup G(S)})^{n} \circ G\left(S^{\bullet}\right)$, where $S^{\bullet}=S$ if $n$ is even, and $S^{\bullet}=R$ if $n$ is odd.

(ii) $R+S \subseteq(F(R)+F(S)) \circ \overline{R \cup S} \circ(G(R)+G(S))$

$$
\subseteq(F(R)+F(S)) \circ R \circ S \circ(G(R)+G(S))
$$

$$
R^{n+1} \subseteq(F(R))^{n} \circ R \circ(G(R))^{n}
$$

$$
R^{*} \subseteq(F(R))^{*} \circ R \circ(G(R))^{*}
$$


(v)

$\left(\operatorname{vi}^{\prime}\right) \quad\left(F(R)+F\left(R^{-}\right)\right) \circ R \circ\left(G(R)+G\left(R^{-}\right)\right)=$

$$
\left(F(R)+F\left(R^{-}\right)\right) \circ R^{-} \circ\left(G(R)+G\left(R^{-}\right)\right)
$$

(vii)

$$
\begin{gathered}
R+S^{-} \subseteq\left(F(R)+F\left(S^{-}\right)\right) \circ \overline{R \cup S} \circ\left(G(R)+G\left(S^{-}\right)\right) \subseteq \\
\left(F(R)+F\left(S^{-}\right)\right) \circ R \circ S \circ\left(G(R)+G\left(S^{-}\right)\right) \\
C g(R) \subseteq\left(F(R)+F\left(R^{-}\right)\right) \circ R \circ\left(G(R)+G\left(R^{-}\right)\right)
\end{gathered}
$$

(x) $C g\left(R_{1} \cup R_{2} \cup \cdots \cup R_{n}\right) \subseteq$

$$
\begin{gathered}
\left(F\left(R_{1}\right)+F\left(R_{1}^{-}\right)+F\left(R_{2}\right)+F\left(R_{2}^{-}\right)+\cdots+F\left(R_{n}\right)+F\left(R_{n}^{-}\right)\right) \circ \\
\frac{R_{1} \cup R_{2} \cup \cdots \cup R_{n}}{} \\
\left(G\left(R_{1}\right)+G\left(R_{1}^{-}\right)+G\left(R_{2}\right)+G\left(R_{2}^{-}\right)+\cdots+G\left(R_{n}\right)+G\left(R_{n}^{-}\right)\right)
\end{gathered}
$$

If $F\left(R_{i}\right) \subseteq R_{i}$ and $G\left(R_{i}\right) \subseteq G_{i}$ for all $i$, then we get equality in (ix); and, similarly, we get equality in (ii), (iv) and (vii) under the corresponding assumptions.

If $F\left(R_{i}\right) \subseteq C g\left(R_{i}\right), F\left(R_{i}^{-}\right) \subseteq C g\left(R_{i}\right), G\left(R_{i}\right) \subseteq C g\left(G_{i}\right)$ and $G\left(R_{i}^{-}\right) \subseteq$ $C g\left(G_{i}\right)$ for all $i$, then we get equality in $(x)$. Similarly, we get equality in (viii) under the corresponding assumptions.

Proof. (i) is the particular case of Theorem 2.2 in which $R_{1}=R_{3}=R_{5}=$ $\cdots=R, R_{2}=R_{4}=R_{6}=\cdots=S$. A direct proof of (i) can be found in [14, Part III].

(ii) is immediate from (i).

(iii) and (iv) are the particular cases $S=R$ of, respectively, (i) and (ii). Notice that, for $n=0$, (iii) becomes the trivial inclusion $R \subseteq R$.

(v) If $a R b$, then $a F(R) t(a, b, b) R^{-} t(a, a, b) G(R) b$.

(vi) follows from (v), applied with $R^{-}$in place of $R$, since $R^{--}=R$.

$\left(\mathrm{vi}^{\prime}\right)$ is immediate from $(\mathrm{v})$ and $(\mathrm{vi})$.

(vii) By (vi) with $S$ in place of $R$ we get $S^{-} \subseteq F\left(S^{-}\right) \circ S \circ G\left(S^{-}\right)$. Hence, $R \cup S^{-} \subseteq F\left(S^{-}\right) \circ(R \cup S) \circ G\left(S^{-}\right)$, and $\overline{R \cup S^{-}} \subseteq F\left(S^{-}\right) \circ \overline{R \cup S} \circ G\left(S^{-}\right)$, since $F\left(S^{-}\right)$and $G\left(S^{-}\right)$are admissible.

By (ii) with $S^{-}$in place of $S$, we get $R+S^{-} \subseteq\left(F(R)+F\left(S^{-}\right)\right) \circ$ $\overline{R \cup S^{-}} \circ\left(G(R)+G\left(S^{-}\right)\right) \subseteq\left(F(R)+F\left(S^{-}\right)\right) \circ F\left(S^{-}\right) \circ \overline{R \cup S} \circ G\left(S^{-}\right) \circ$ $\left(G(R)+G\left(S^{-}\right)\right)=\left(F(R)+F\left(S^{-}\right)\right) \circ \overline{R \cup S} \circ\left(G(R)+G\left(S^{-}\right)\right)$.

(viii) is immediate from (vii), since $C g(R)=R+R^{-}$, since $R$ is admissible. 
(ix) follows from Theorem 2.2 in a way similar to (i), since if $(a, b) \in R_{1}+$ $R_{2}+\cdots+R_{n}$ then $(a, b) \in R_{1} \circ R_{2} \cdots \circ R_{n} \circ R_{1} \circ R_{2} \cdots \circ R_{n} \circ R_{1} \circ R_{2} \cdots \circ R_{n} \circ \ldots$

(x) Since $C g\left(R_{1} \cup R_{2} \cup \cdots \cup R_{n}\right)=R_{1}+R_{1}^{-}+R_{2}+R_{2}^{-}+\cdots+R_{n}+R_{n}^{-}$, we get, by applying (ix):

$$
\begin{aligned}
& C g\left(R_{1} \cup R_{2} \cup \cdots \cup R_{n}\right) \subseteq \\
& \left(F\left(R_{1}\right)+F\left(R_{1}^{-}\right)+F\left(R_{2}\right)+F\left(R_{2}^{-}\right)+\cdots+F\left(R_{n}\right)+F\left(R_{n}^{-}\right)\right) \circ \\
& \quad \frac{R_{1} \cup R_{1}^{-} \cup R_{2} \cup R_{2}^{-} \cup \cdots \cup R_{n} \cup R_{n}^{-}}{} \circ \\
& \left(G\left(R_{1}\right)+G\left(R_{1}^{-}\right)+G\left(R_{2}\right)+G\left(R_{2}^{-}\right)+\cdots+G\left(R_{n}\right)+G\left(R_{n}^{-}\right)\right) .
\end{aligned}
$$

By (vi) we get $R_{i}^{-} \subseteq F\left(R_{i}^{-}\right) \circ R_{i} \circ G\left(R_{i}^{-}\right)$, for all $i=1, \ldots, n$. Hence, $R_{1} \cup R_{1}^{-} \cup R_{2} \cup R_{2}^{-} \cup \cdots \cup R_{n} \cup R_{n}^{-} \subseteq\left(F\left(R_{1}^{-}\right)+F\left(R_{2}^{-}\right)+\cdots+F\left(R_{n}^{-}\right)\right) \circ$ $\left(R_{1} \cup R_{2} \cup \cdots \cup R_{n}\right) \circ\left(G\left(R_{1}^{-}\right)+G\left(R_{2}^{-}\right)+\cdots+G\left(R_{n}^{-}\right)\right)$. Now $(\mathrm{x})$ follows as in the proof of (vii).

The last two statements are trivial, since the $R_{i}$ 's are reflexive and admissible.

\section{Terms Mal'cev throughout a Variety}

We shall also be interested in the case when $F$ and $G$ are defined globally on all algebras of some variety.

If $\mathcal{V}$ is a variety, let us say that $F$ is a global operator on $\mathcal{V}$ for admissible and reflexive relations if and only if to any algebra $\mathbf{A} \in \mathcal{V} F$ assigns an operation $F_{\mathbf{A}}: \operatorname{Adm}(\mathbf{A}) \rightarrow \operatorname{Adm}(\mathbf{A})$. In case there is no danger of confusion, we shall omit the subscript $\mathbf{A}$.

We say that a global operator on $\mathcal{V}$ satisfies the homomorphism property if and only if whenever $\mathbf{A}, \mathbf{B} \in \mathcal{V}, \phi: \mathbf{B} \rightarrow \mathbf{A}$ is a homomorphism, and $R \in \operatorname{Adm}(\mathbf{B})$ then $\phi\left(F_{\mathbf{B}}(R)\right) \subseteq F_{\mathbf{A}}(\phi(R))$. Here, $\phi(R)$ denotes the smallest admissible and reflexive relation on $\mathbf{A}$ which contains $\{(\phi(b), \phi(c)) \mid b R c\}$.

As noticed in [12, Part II, Remark 2.5], essentially all commutators defined using matrices satisfy the homomorphism property $\phi([\alpha, \beta]) \subseteq[\phi(\alpha), \phi(\beta)]$. A related homomorphism property can be used in order to characterize the commutator in congruence modular varieties (see [2, Definition 3.1 and Theorem 4.10]). In [10, Definition 3.4] and [14] we have defined commutator operations for reflexive and admissible relations. Propositions 3.2 and 3.3 below show that these commutators (and many more) satisfy the homomorphism property $\phi([R, S]) \subseteq[\phi(R), \phi(S)]$, hence the next Theorem can be applied, for example, in the case $F(R)=G(R)=[R, R]$, where [,] is one of the commutators introduced in [14].

As usual, $F$ is said to be monotone if and only if $F(R) \subseteq F(S)$ whenever $R \subseteq S$.

Theorem 3.1. Suppose that $\mathcal{V}$ is a variety, $F, G$ are global operators on $\mathcal{V}$ for admissible and reflexive relations, $F, G$ are monotone and satisfy the homomorphism property. Then the following are equivalent: 
(i) $\mathcal{V}$ has a term which is Mal'cev modulo $F_{\mathbf{A}}$ and $G_{\mathbf{A}}$ for every algebra A in $\mathcal{V}$.

(ii) Every $\mathbf{A} \in \mathcal{V}$ has a term which is Mal'cev modulo $F_{\mathbf{A}}$ and $G_{\mathbf{A}}$.

(iii) The free algebra $\mathbf{X}$ in $\mathcal{V}$ generated by 2 elements has a term which is Mal'cev modulo $F_{\mathbf{X}}$ and $G_{\mathbf{X}}$.

(iv) In the free algebra $\mathbf{X}$ in $\mathcal{V}$ generated by the two elements $x, y$ there is a ternary term $t$ such that, if $S$ is the smallest admissible and reflexive relation of $\mathbf{X}$ containing $(x, y)$, then

$$
x F_{\mathbf{X}}(S) t(x, y, y) \quad \text { and } \quad t(x, x, y) G_{\mathbf{X}}(S) y .
$$

(v) In every algebra $\mathbf{A} \in \mathcal{V}$ and for every relation $R \in \operatorname{Adm}(\mathbf{A})$, the following holds:

$$
R \circ R \subseteq F_{\mathbf{A}}(R) \circ R \circ G_{\mathbf{A}}(R) .
$$

(vi) In the free algebra $\mathbf{X}$ in $\mathcal{V}$ generated by 3 elements the following holds:

$$
R \circ R \subseteq F_{\mathbf{X}}(R) \circ R \circ G_{\mathbf{X}}(R),
$$

for every relation $R \in \operatorname{Adm}(\mathbf{X})$.

(vii) In the free algebra $\mathbf{X}$ in $\mathcal{V}$ generated by the three elements $x, y, z$, the following holds, where $S$ is the smallest admissible and reflexive relation of $\mathbf{X}$ containing both $(x, y)$ and $(y, z)$ :

$$
S \circ S \subseteq F_{\mathbf{X}}(S) \circ S \circ G_{\mathbf{X}}(S)
$$

(viii) In every algebra $\mathbf{A} \in \mathcal{V}$ and for every relation $R \in \operatorname{Adm}(\mathbf{A})$, the following holds:

$$
R \subseteq F_{\mathbf{A}}(R) \circ R^{-} \circ G_{\mathbf{A}}(R)
$$

(ix) In the free algebra $\mathbf{X}$ in $\mathcal{V}$ generated by 2 elements the following holds:

$$
R \subseteq F_{\mathbf{X}}(R) \circ R^{-} \circ G_{\mathbf{X}}(R),
$$

for every relation $R \in \operatorname{Adm}(\mathbf{X})$.

(x) In the free algebra $\mathbf{X}$ in $\mathcal{V}$ generated by the two elements $x, y$ the following holds, where $S$ is the smallest admissible and reflexive relation of $\mathbf{X}$ containing $(x, y)$ :

$$
S \subseteq F_{\mathbf{X}}(S) \circ S^{-} \circ G_{\mathbf{X}}(S) .
$$

Proof. (i) $\Rightarrow$ (ii) $\Rightarrow$ (iii) $\Rightarrow$ (iv) are trivial.

(iv) $\Rightarrow$ (i) We claim that the term $t$ given by (iv) is Mal'cev modulo $F_{\mathbf{A}}$ and $G_{\mathbf{A}}$, for every algebra $\mathbf{A}$ in $\mathcal{V}$.

Indeed, suppose that $\mathbf{A}$ in $\mathcal{V}, a, b \in \mathbf{A}, R \in \operatorname{Adm}(\mathbf{A})$, and $a R b$.

Since $\mathbf{X}$ is the free algebra in $\mathcal{V}$ generated by $\{x, y\}$, there is a homomorphism $\phi: \mathbf{X} \rightarrow \mathbf{A}$ such that $\phi(x)=a$ and $\phi(y)=b$, and hence $\phi(t(x, y, y))=t(a, b, b), \phi(t(x, x, y))=t(a, a, b)$.

Since $x F_{\mathbf{X}}(S) t(x, y, y)$, we have $\phi(x) \phi\left(F_{\mathbf{X}}(S)\right) \phi(t(x, y, y))$, hence, by the homomorphism property, a $F_{\mathbf{A}}(\phi(S)) t(a, b, b)$.

Since $S$ is the admissible and reflexive relation generated by $(x, y)$, and $\phi$ is a homomorphism, then $\phi(S)$ is the admissible and reflexive relation generated by $(\phi(x), \phi(y))=(a, b)$, and, since $a R b$, we have that $\phi(S) \subseteq R$; 
thus, by the monotonicity of $F_{\mathbf{A}}$, we get $F_{\mathbf{A}}(\phi(S)) \subseteq F_{\mathbf{A}}(R)$ and, eventually, $a F_{\mathbf{A}}(R) t(a, b, b)$.

Exactly in the same way, we get $t(a, a, b) G_{\mathbf{A}}(R) b$, thus $t$ is Mal'cev modulo $F$ and $G$ for every algebra in $\mathcal{V}$.

Having proved that (iv) $\Rightarrow$ (i), we have that (i)-(iv) are all equivalent.

(i) $\Rightarrow$ (v) is from Lemma 2.1(iv).

(v) $\Rightarrow$ (vi) $\Rightarrow$ (vii) are trivial.

(vii) $\Rightarrow$ (i). Since $x S y S z$, we have $(x, z) \in S \circ S$, hence $(x, z) \in F_{\mathbf{X}}(S) \circ$ $S \circ G_{\mathbf{X}}(S)$, by assumption. This means that $\mathbf{X}$ has terms $t_{1}(x, y, z)$ and $t_{2}(x, y, z)$ such that $\left(x, t_{1}(x, y, z)\right) \in F_{\mathbf{X}}(S),\left(t_{1}(x, y, z), t_{2}(x, y, z)\right) \in S$ and $\left(t_{2}(x, y, z), z\right) \in G_{\mathbf{X}}(S)$.

Notice that $S=\{(u(x, y, z, x, y), u(x, y, z, y, z)) \mid u$ a term of $\mathbf{X}\}$, since the right-hand relation is reflexive, admissible, and contains $(x, y)$ and $(y, z)$; moreover, every other reflexive admissible relation containing $(x, y)$ and $(y, z)$ contains all pairs of the form $(u(x, y, z, x, y), u(x, y, z, y, z))$. Hence, $\left(t_{1}(x, y, z), t_{2}(x, y, z)\right) \in S$ means that $\mathbf{X}$ has a 5 -ary term $t^{\prime}$ such that $t_{1}(x, y, z)=t^{\prime}(x, y, z, x, y)$ and $t^{\prime}(x, y, z, y, z)=t_{2}(x, y, z)$.

We claim that the ternary term $t(x, y, z)=t^{\prime}(x, y, z, x, z)$ is Mal'cev modulo $F$ and $G$ throughout $\mathcal{V}$. In order to prove it, first notice that $t_{1}(x, y, y)=t(x, y, y)$, and $t(y, y, z)=t_{2}(y, y, z)$. Notice that, since $\mathbf{X}$ is a free algebra, the above identities hold throughout $\mathcal{V}$.

Suppose that $\mathbf{A} \in \mathcal{V}, a, b \in \mathbf{A}, R \in \operatorname{Adm}(\mathbf{A})$, and $a R b$. There is a homomorphism $\phi: \mathbf{X} \rightarrow \mathbf{A}$ such that $\phi(x)=a, \phi(y)=b$, and $\phi(z)=b$, hence $\phi\left(t_{1}(x, y, y)\right)=t_{1}(a, b, b)$.

Since $x F_{\mathbf{X}}(S) t_{1}(x, y, z)$, we have $\phi(x) \phi\left(F_{\mathbf{X}}(S)\right) \phi\left(t_{1}(x, y, z)\right)$, hence, by the homomorphism property, $a F_{\mathbf{A}}(\phi(S)) t_{1}(a, b, b)$.

Since $S$ is the admissible and reflexive relation generated by $(x, y),(y, z)$, then $\phi(S)$ is the admissible and reflexive relation generated by $(\phi(x), \phi(y))$, $(\phi(y), \phi(z))$, that is, generated by $(a, b),(b, b)$, hence generated simply by $(a, b)$. Since $a R b$, we have that $\phi(S) \subseteq R$; thus, by the monotonicity of $F_{\mathbf{A}}$, we get $F_{\mathbf{A}}(\phi(S)) \subseteq F_{\mathbf{A}}(R)$ and, eventually, $a F_{\mathbf{A}}(R) t_{1}(a, b, b)=t(a, b, b)$.

Exactly in the same way, by considering the homomorphism $\psi: \mathbf{X} \rightarrow$ A satisfying $\psi(x)=a, \psi(y)=a$, and $\psi(z)=b$, we get $t(a, a, b)=$ $t_{2}(a, a, b) G_{\mathbf{A}}(R) b$.

Thus $t$ is Mal'cev modulo $F$ and $G$ for every algebra in $\mathcal{V}$, and we have closed our second cycle of equivalencies.

(i) $\Rightarrow$ (viii) follows from Corollary $2.3(\mathrm{v})$.

(viii) $\Rightarrow$ (ix) $\Rightarrow$ (x) are trivial.

$(\mathrm{x}) \Rightarrow$ (iv). Since $x S y$, then, by assumption, $(x, y) \in F_{\mathbf{X}}(S) \circ S^{-} \circ G_{\mathbf{X}}(S)$. This means that there are binary terms $t_{1}$ and $t_{2}$ such that $\left(x, t_{1}(x, y)\right) \in$ $F_{\mathbf{X}}(S),\left(t_{1}(x, y), t_{2}(x, y)\right) \in S^{-}$, and $\left(t_{2}(x, y), y\right) \in G_{\mathbf{X}}(S)$.

That $\left(t_{1}(x, y), t_{2}(x, y)\right) \in S^{-}$means that there is a ternary term $t$ such that $t_{1}(x, y)=t(x, y, y)$ and $t(x, x, y)=t_{2}(x, y)$, since $S=\{(u(x, x, y)$, $u(x, y, y)) \mid u$ a term of $\mathbf{X}\}$ (cf., e.g., the proof of Theorem 1 (vi) $\Rightarrow$ (vii) in $[14$, Part II], or the proof of (vii) $\Rightarrow$ (i) above). 
Thus, $x F_{\mathbf{X}}(S) t_{1}(x, y)=t(x, y, y), t(x, x, y)=t_{2}(x, y) G_{\mathbf{X}}(S) y$, that is, the hypotheses of (iv) are satisfied.

In the particular case when $F(R)=G(R)=0$ constantly, Theorem 3.1 gives, via Mal'cev's Theorem 1.1, the well known result that a variety $\mathcal{V}$ is congruence permutable if and only if $R \circ R \subseteq R$ holds in $\mathcal{V}$ for admissible and reflexive relations, if and only if $R \subseteq R^{-}$holds in $\mathcal{V}$ for admissible and reflexive relations.

Theorem 3.1 generalizes Theorem $2.2(\mathrm{i}) \Leftrightarrow(\mathrm{ii}) \Leftrightarrow($ ii'), Remark 2.4 and parts of Theorem 3.2 in [12, Part II]. Notice that, for example, condition (viii) in Theorem 3.1 (for $F(R)=G(R)=[C g(R), C g(R)]$ ) implies condition (vii) in [12, Part II, Theorem 3.2]. However, the equivalence of certain conditions in [12, Part II, Theorem 3.2] depends on the actual definitions of the commutators dealt there.

We now show that the commutators defined in $[10,14]$ satisfy the homomorphism property. Actually, we shall deal with $n$-ary global operators on $\mathcal{V}$, that is, operators depending on $n$-variables (as above, each variable is intended to be an admissible and reflexive relation). In this general situation, the homomorphism property reads $\phi\left(F_{\mathbf{B}}\left(R_{1}, \ldots, R_{n}\right)\right) \subseteq$ $F_{\mathbf{A}}\left(\phi\left(R_{1}\right), \ldots, \phi\left(R_{n}\right)\right)$, for every $\mathbf{A}, \mathbf{B} \in \mathcal{V}$, homomorphism $\phi: \mathbf{B} \rightarrow \mathbf{A}$, and $R_{1}, \ldots, R_{n} \in \operatorname{Adm}(\mathbf{B})$. Moreover, $F$ is monotone if and only if $R_{1} \subseteq S_{1}$, $\ldots, R_{n} \subseteq S_{n}$ imply $F\left(R_{1}, \ldots, R_{n}\right) \subseteq F\left(S_{1}, \ldots, S_{n}\right)$.

If $\mathbf{A}$ is any algebra, and $R, S$ are compatible and reflexive relations, $M(R, S)$ is defined to be the set of all matrices of the form

$$
\left|\begin{array}{ll}
t(\bar{a}, \bar{b}) & t\left(\bar{a}, \bar{b}^{\prime}\right) \\
t\left(\bar{a}^{\prime}, \bar{b}\right) & t\left(\bar{a}^{\prime}, \bar{b}^{\prime}\right)
\end{array}\right|
$$

where $\bar{a}, \bar{a}^{\prime} \in A^{h}, \bar{b}, \bar{b}^{\prime} \in A^{k}$, for some $h, k \geq 0, t$ is an $h+k$-ary term operation of $\mathbf{A}$, and $\bar{a} R \bar{a}^{\prime}, \bar{b} S \bar{b}^{\prime}$. Here, $\bar{a} R \bar{a}^{\prime}$ means $a_{1} R a_{1}^{\prime}, a_{2} R a_{2}^{\prime}, \ldots$

For $R, S, T, U$ compatible and reflexive relations, let

$$
K(R, S ; U ; T)=\left\{(z, w)\left|\begin{array}{cc}
x & y \\
z & w
\end{array}\right| \in M(R, S), x U z, x T y,\right\} .
$$

$K(R, S ; 1 ; T)$ is denoted by $K(R, S ; T)$ in [14], and various commutators for relations are constructed there from $K(R, S ; T)$.

Proposition 3.2. For every variety $\mathcal{V}, K(R, S ; U ; T)$ and $K(R, S ; T)$ are monotone global operators on $\mathcal{V}$ satisfying the homomorphism property.

Proof. We shall give the proof for $K(R, S ; U ; T)$. The proof for $K(R, S ; T)$ is similar and simpler (and, anyway, is the particular case $U=1$ constantly). The operators are trivially monotone.

We now prove that $K(R, S ; U ; T)$ satisfies the homomorphism property. Suppose that $\mathbf{A}, \mathbf{B} \in \mathcal{V}, \phi: \mathbf{B} \rightarrow \mathbf{A}, R, S, T, U \in \operatorname{Adm}(\mathbf{B}), x^{\prime}, w^{\prime} \in \mathbf{A}$, and $\left(x^{\prime}, w^{\prime}\right) \in \phi(K(R, S ; U ; T))$. We have to show that $\left(x^{\prime}, w^{\prime}\right) \in K(\phi(R), \phi(S)$; $\phi(U) ; \phi(T))$. 
That $\left(x^{\prime}, w^{\prime}\right) \in \phi(K(R, S ; U ; T))$ means that $\mathbf{B}$ has couples $\left(x_{1}, w_{1}\right), \ldots$, $\left(x_{m}, w_{m}\right) \in K(R, S ; U ; T)$ such that, in $\mathbf{A},\left(x^{\prime}, w^{\prime}\right)$ belongs to the admissible and reflexive relation generated by $\left(\phi\left(x_{1}\right), \phi\left(w_{1}\right)\right), \ldots,\left(\phi\left(x_{m}\right), \phi\left(w_{m}\right)\right)$. This means that $\mathbf{A}$ has an $m$-ary polynomial $p$ such that $x^{\prime}=p\left(\phi\left(x_{1}\right), \ldots, \phi\left(x_{m}\right)\right)$, and $w^{\prime}=p\left(\phi\left(w_{1}\right), \ldots, \phi\left(w_{m}\right)\right)$.

Since $\left(x_{i}, w_{i}\right) \in K(R, S ; U ; T)$ for every $i=1, \ldots m$, then, by the definition of $K(R, S ; U ; T)$, there are matrices

$$
\left|\begin{array}{cc}
t_{i}\left(\bar{a}_{i}, \bar{b}_{i}\right) & t_{i}\left(\bar{a}_{i}, \bar{b}_{i}^{\prime}\right) \\
t_{i}\left(\bar{a}_{i}^{\prime}, \bar{b}_{i}\right) & t_{i}\left(\bar{a}_{i}^{\prime}, \bar{b}_{i}^{\prime}\right)
\end{array}\right|
$$

such that $\bar{a}_{i} R \bar{a}_{i}^{\prime}, \bar{b}_{i} S \bar{b}_{i}^{\prime}, t_{i}\left(\bar{a}_{i}, \bar{b}_{i}\right) T t_{i}\left(\bar{a}_{i}, \bar{b}_{i}^{\prime}\right), t_{i}\left(\bar{a}_{i}, \bar{b}_{i}\right) U t_{i}\left(\bar{a}_{i}^{\prime}, \bar{b}_{i}\right)$, and $x_{i}=$ $t_{i}\left(\bar{a}_{i}^{\prime}, \bar{b}_{i}\right), w_{i}=t_{i}\left(\bar{a}_{i}^{\prime}, \bar{b}_{i}^{\prime}\right)$.

For sake of brevity, let us write $t_{i}\left(\phi \bar{a}_{i}, \phi \bar{b}_{i}\right)$ in place of $t_{i}\left(\phi\left(a_{i 1}\right), \phi\left(a_{i 2}\right), \ldots\right.$, $\left.\phi\left(b_{i 1}\right), \phi\left(b_{i 2}\right), \ldots\right)$. The matrix

$$
\left|\begin{array}{ll}
p\left(t_{1}\left(\phi \bar{a}_{1}, \phi \bar{b}_{1}\right), \ldots, t_{m}\left(\phi \bar{a}_{m}, \phi \bar{b}_{m}\right)\right) & p\left(t_{1}\left(\phi \bar{a}_{1}, \phi \bar{b}_{1}^{\prime}\right), \ldots, t_{m}\left(\phi \bar{a}_{m}, \phi \bar{b}_{m}^{\prime}\right)\right) \\
p\left(t_{1}\left(\phi \bar{a}_{1}^{\prime}, \phi \bar{b}_{1}\right), \ldots, t_{m}\left(\phi \bar{a}_{m}^{\prime}, \phi \bar{b}_{m}\right)\right) & p\left(t_{1}\left(\phi \bar{a}_{1}^{\prime}, \phi \bar{b}_{1}^{\prime}\right), \ldots, t_{m}\left(\phi \bar{a}_{m}^{\prime}, \phi \bar{b}_{m}^{\prime}\right)\right)
\end{array}\right|
$$

belongs to $M(\phi(R), \phi(S))$; moreover, since $\phi$ is a homomorphism, the above matrix equals

$$
\left|\begin{array}{ll}
p\left(\phi\left(t_{1}\left(\bar{a}_{1}, \bar{b}_{1}\right)\right), \ldots, \phi\left(t_{m}\left(\bar{a}_{m}, \bar{b}_{m}\right)\right)\right) & p\left(\phi\left(t_{1}\left(\bar{a}_{1}, \bar{b}_{1}^{\prime}\right)\right), \ldots, \phi\left(t_{m}\left(\bar{a}_{m}, \bar{b}_{m}^{\prime}\right)\right)\right) \\
p\left(\phi\left(t_{1}\left(\bar{a}_{1}^{\prime}, \bar{b}_{1}\right)\right), \ldots, \phi\left(t_{m}\left(\bar{a}_{m}^{\prime}, \bar{b}_{m}\right)\right)\right) & p\left(\phi\left(t_{1}\left(\bar{a}_{1}^{\prime}, \bar{b}_{1}^{\prime}\right)\right), \ldots, \phi\left(t_{m}\left(\bar{a}_{m}^{\prime}, \bar{b}_{m}^{\prime}\right)\right)\right)
\end{array}\right|
$$

Since $t_{i}\left(\bar{a}_{i}, \bar{b}_{i}\right) T t_{i}\left(\bar{a}_{i}, \bar{b}_{i}^{\prime}\right)$, and since $T$ is admissible, the elements in the upper row of the matrix are $\phi(T)$-related; similarly, the elements in the lefthand column are $\phi(U)$-related. Since $x_{i}=t_{i}\left(\bar{a}_{i}^{\prime}, \bar{b}_{i}\right)$, and $w_{i}=t_{i}\left(\bar{a}_{i}^{\prime}, \bar{b}_{i}^{\prime}\right)$, then the second row of the matrix consists of the elements $p\left(\phi\left(x_{1}\right), \ldots, \phi\left(x_{m}\right)\right)=$ $x^{\prime}$, and $p\left(\phi\left(w_{1}\right), \ldots, \phi\left(w_{m}\right)\right)=w^{\prime}$. This means that the matrix witnesses that $\left(x^{\prime}, w^{\prime}\right) \in K(\phi(R), \phi(S) ; \phi(U) ; \phi(T))$.

Proposition 3.3. If $\mathcal{V}$ is a variety, $F_{1}, F_{2}, \ldots$ are $n$-ary global operators on $\mathcal{V}$ for admissible and reflexive relations, and $F_{1}, F_{2}, \ldots$ satisfy the homomorphism property, then so do the following operators:

(i) $G_{1}(\bar{R})=F_{1}(\bar{R}) \circ F_{2}(\bar{R})$;

(ii) $G_{2}(\bar{R})=F_{1}(\bar{R}) \cap F_{2}(\bar{R})$;

(iii) $G_{3}(\bar{R})=\overline{F_{1}(\bar{R}) \cup F_{2}(\bar{R})}$;

(iv) $G_{4}(\bar{R})=F_{1}(\bar{R})+F_{2}(\bar{R})$;

(v) $G_{5}(\bar{R})=\left(F_{1}(\bar{R})\right)^{*}$;

(vi) $G_{6}(\bar{R})=K\left(F_{1}(\bar{R}), F_{2}(\bar{R}) ; F_{3}(\bar{R}) ; F_{4}(\bar{R})\right)$.

(vii) $G_{7}(\bar{R})=K\left(F_{1}(\bar{R}), F_{2}(\bar{R}) ; F_{4}(\bar{R})\right)$.

If, in addition, $F_{1}, F_{2}, \ldots$ are monotone, then so are $G_{1}, \ldots, G_{7}$.

Proof. The proof is similar to the proof of Proposition 3.2, in many cases simpler.

Since, trivially, the constant operators $F(\bar{R})=0$ and $F(\bar{R})=1$, the operator $F(R)=R^{-}$, as well as the projections, are monotone and satisfy the homomorphism property, we get from Proposition 3.3(vii)(v) that, for 
example, $F(R, S)=K(R, S ; 0)^{*}$ is monotone and satisfies the homomorphism property. Similarly, Proposition 3.3 can be iterated in order to show that all the commutators introduced in [14] are monotone and satisfy the homomorphism property. Notice also that condition (vi) in Proposition 3.3 is more general than Proposition 3.2: just take $n=4$, and let each $F_{i}$ be the projection onto the $i$-th coordinate.

\section{REFERENCES}

[1] G. Czédli, E. Horváth, P. Lipparini, Optimal Mal'tsev conditions for congruence modular varieties, Algebra Universalis 53 (2005), no. 2-3, 267-279.

[2] Ralph Freese and Ralph McKenzie, Commutator theory for congruence modular varieties, London Mathematical Society Lecture Note Series, vol. 125, Cambridge University Press, Cambridge, 1987.

[3] H. Peter Gumm, Geometrical methods in congruence modular algebras, Memoirs of the American Mathematical Society 45 (1983), no. 286, viii+79.

[4] David Hobby and Ralph McKenzie, The structure of finite algebras, Contemporary Mathematics, vol. 76, American Mathematical Society, Providence, RI, 1988.

[5] B. Jónsson, Congruence varieties, Algebra Universalis, 10 (1980), 355-394.

[6] K. A. Kearnes, Varieties with a difference term, J. Algebra 177 (1995), no. 3, 926-960.

[7] K. A. Kearnes, E. W. Kiss, The triangular principle is equivalent to the triangular scheme, Algebra Universalis 54 (2005), no. 3, 273-283.

[8] http://www.cs.elte.hu/ ewkiss/papers/congidSzeged2005.pdf

[9] Paolo Lipparini, Commutator theory without join-distributivity, Transactions of the American Mathematical Society 346 (1994), no. 1, 177-202.

[10] Congruence identities satisfied in n-permutable varieties, Bollettino U. M. I. (7) 8-B (1994), 851-868.

[11] _ n-permutable varieties satisfy nontrivial congruence identities, Algebra Universalis 33 (1995), no. 2, 159-168.

[12] _ A characterization of varieties with a difference term Canadian Mathematical Bulletin. Bulletin Canadien de Mathématiques 39 (1996), no. 3, 308-315; II. Neutral $=$ meet semi-distributive, 41 (1998), no. 3, 318-327.

[13] _ A Kiss 4-difference term from a ternary term, Algebra Universalis 42 (1999), no. $1-2,153-154$.

[14] Towards commutator theory for relations, http://arxiv.org/math.GM/0507039; II, http://arxiv.org/math.GM/0509233; III, http://arxiv.org/math.GM/0511313 (2005).

[15] _ Every m-permutable variety satisfies the congruence identity $\alpha \beta_{h}=\alpha \gamma_{h}$, accepted by Proceedings of the American Mathematical Society.

[16] A. I. Mal'cev, On the general theory of algebraic systems, Mat. Sb. N.S., 35(77) (1954), 3-20.

[17] Jonathan D. H. Smith, Mal'cev varieties, Springer-Verlag, Berlin, 1976, Lecture Notes in Mathematics, Vol. 554.

[18] W. Taylor, Some applications of the Term Condition, Algebra Universalis, 14 (1982), 11-24.

Dipartimento di Matematica, Viale della Ricerca Scientifica, il Università

DI Roma (Tor VERGATTA), ROME ITALY

E-mail address: lipparin@axp.mat.uniroma2.it

URL: http://www.mat.uniroma2.it/ lipparin 